\title{
Unsteady particle motion in an acoustic standing wave field
}

\author{
S. Wang ${ }^{\mathrm{a}}$, J. S. Allen ${ }^{\mathrm{b}}$ and A. M. Ardekani ${ }^{\mathrm{a}}$ \\ ${ }^{a}$ School of Mechanical Engineering, Purdue University, West Lafayette, IN, USA; ${ }^{\mathrm{b}}$ Department of \\ Mechanical Engineering, University of Hawaii-Manoa, Honolulu, HI, USA
}

\begin{abstract}
The acoustic-based separation has attracted considerable attention in biomedical research, such as sorting of cells and particles. Current design principles used for acoustic systems are based on the steady Stokes theory, equating the Stokes drag with the primary radiation force. However, this approach is not valid for large cells/particles or in the presence of particle-particle interaction. In this work, we analytically examine unsteady inertial affects and particle-particle hydrodynamic interaction on the particle motion in a viscous fluid in the presence of an acoustic standing wave field. Comparing our results to the steady Stokes theory, we find that the unsteady inertial force decreases the particle's velocity, while particle-particle interaction enhances it. For a particular acoustic-based separation approach 'tilted-angle standing surface acoustic waves (taSSAW)', we find that both effects of unsteady inertial force and particle-particle interaction are evident and should be considered for $O(10 \mu \mathrm{m})$ particles or larger. Our study improves the current predictions of particle trajectory in acoustic-based separation devices.
\end{abstract}

\section{ARTICLE HISTORY}

Received 31 January 2017

Accepted 9 March 2017

\section{KEYWORDS}

Particle motion; low Reynolds hydrodynamics; unsteady inertial effects; acoustic-based separation

\section{Introduction}

Many underlying ideas and concepts associated with acoustic-based separation have been known and investigated for several decades, but widespread commercial usage have not yet been achieved (Coakley, Whitworth, Grundy, Gould, \& Allman, 1994; Johnson \& Feke, 1995; Yeo \& Friend, 2009, 2011). Acoustic-based separation focuses on the sorting/isolating specific cells/particles from different samples. Recently, acoustic separation of circulating tumour cells (CTCs) has been studied (Ding et al., 2014; Guo et al., 2016; Li et al., 2015). CTCs have become the established liquid biopsy target for cancer diagnosis and prognosis (Plaks, Koopman, \& Werb, 2013). The selection of CTCs requires high-throughput separation techniques due to their extremely low concentration in the peripheral blood. Acoustic-based cell separation methods in microfluidic

CONTACT A.M. Ardekani $\otimes$ ardekani@purdue.edu

${ }^{1}$ Davidson School of Chemical Engineering, Purdue University, 480 Stadium Mall Drive, West Lafayette, Indiana 47907 
devices are a potential way to distinguish cells based on both their geometry and physical properties. Although acoustic-based separation techniques have been progressively improved (Li et al., 2015), their efficacy has remained low for large cells and high-throughput situations.

Tilted-angle standing surface acoustic waves (taSSAW) separation strategies have been applied to study blood samples obtained from cancer patients (Li et al., 2015). The design of optimum devices is based on the steady Stokes theory, which neglects both unsteady inertial effects and hydrodynamic interactions due to the presence of relatively large particles and high-throughput analysis, respectively. Nevertheless, the advances in microfluidics have fostered the development of more precise acoustic resonator systems needed for particles of cellular size. More in-depth theoretical and numerical treatments are sought for the design of acoustic resonator systems.

For acoustic-based separation, the particle motion is the result of primary radiation force based on its specific physical properties. The primary radiation force originates from the scattering interaction between the particles and the incident sound field. The theory of acoustic radiation force follows from the early work by King (1934) which considered incompressible particles. For particle sizes much smaller than the acoustic wavelength, incompressible formulations can be used to study particle trajectories. For these spatial scales, the flow field in the close proximity of the particle is approximated as incompressible. These assumptions have been the basis for many modelling efforts for evaluating particle trajectories (Settnes \& Bruus, 2012).

The importance of the unsteady force on the particle motion was first described by Temkin and Leung (1976), Temkin (1981, 2005) who illustrated the effects of history force in the Basset-Boussinesq-Oseen (BBO) equation on a spherical particle in an acoustic field. Subsequently, BBO equation has been applied to study the unsteady motion of a particle in an acoustic standing wave (Aboobaker, Blackmore, \& Meegoda, 2005; Allen, Kobayashi, \& Coimbra, 2006), which assumes a constant acoustic force in the long wavelength limit. To date, the problem of the unsteady particle motion accounting for the variation in the acoustic force has remained unresolved, and this study aims at resolving this issue.

The role of unsteady forces on particle trajectories in an acoustic field remains a topic for ongoing research especially with respect to the particle-particle interactions (Bruus, 2012). The variation in the unsteady forces due to the hydrodynamic interaction between particles is currently unknown (Temkin, 2005). In this work, we analytically examine the unsteady motion of rigid, spherical particles suspended in an acoustic standing wave field due to the primary radiation force. By combining both approaches of Laplace transform and convolution, we are able to study particle's motion with the variation of acoustic radiation force. The role of particle-particle interaction in an acoustic standing wave field is further investigated for the unsteady case when two particles are 
moving along their lines of centre and side by side. Since the problem of interest is linear due to small values of particle Reynolds number, the solution to an arbitrary motion of two particles can be obtained from the superposition of these two problems. Finally, we apply our model to the taSSAW-based particle motion to account for the effect of large particle size and particle-particle interaction.

\section{Equation of motion for particles in an acoustic field}

We consider the motion of spherical particles in an acoustic standing wave field. Particles in a viscous fluid are driven by an acoustic force, which is a sum of primary and secondary radiation forces. For the flow acoustophoresis, the primary radiation force is the dominant acoustic force for particle manipulation (Augustsson et al., 2011; Bruus, 2012; Laurell, Petersson, \& Nilsson, 2007). In this case, the influence of secondary acoustic force, which is caused by the reflected sound wave in a particle suspension, is relatively weak (Laurell et al., 2007; Weiser, Apfel, \& Neppiras, 1984). The primary radiation force is given as

$$
F_{a c}^{*}=3 \tilde{V} E_{a c}^{*} K G \sin \left(2 K X^{*}\right),
$$

where $\tilde{V}$ is the volume of the particle, and $E_{a c}^{*}$ is the acoustic energy density. The factor $\sin \left(2 K X^{*}\right)$ captures the variation of the primary radiation force with the spatial coordinate $X^{*}$, where $K=2 \pi / \lambda$ is the wave number of the acoustic radiation force, and $\lambda$ is the wavelength of the acoustic standing wave field. Acoustic contrast factor $G$ determines the direction of the motion of the particle. Rigid particles (Augustsson et al., 2011) and red blood cells (Laurell et al., 2007) have positive $G$ and move towards the pressure nodal line. Particles with negative $G$ values (e.g. lipid capsules (Laurell et al., 2007)) move towards the pressure antinodal line. In this work, we focus on positive $G$ values and consequently, the resultant particle motion is towards the nodal line. A sketch of the primary radiation force is provided in Figure 1(a), where the solid line indicates the nodal line of the acoustic field. We first investigate the motion of a single particle by considering unsteady inertial effects, and subsequently formulate the equation of motion for two-particle configurations.

\subsection{Equation of motion for a single spherical particle}

The equation of motion for a small spherical particle in an acoustical field in a viscous fluid can be written as (Maxey \& Riley, 1983)

$$
\begin{aligned}
m_{p} \frac{\mathrm{d} V^{*}}{\mathrm{~d} t^{*}}= & -6 \pi \mu R V^{*}-\frac{1}{2} m_{f} \frac{\mathrm{d} V^{*}}{\mathrm{~d} t^{*}}-6 \pi \mu R^{2} \int_{0}^{t^{*}} \mathrm{~d} \tau^{*} \frac{\mathrm{d} V^{*} / \mathrm{d} \tau^{*}}{\left[\pi v\left(t^{*}-\tau^{*}\right)\right]^{1 / 2}} \\
& +F_{a c}^{*},
\end{aligned}
$$

where superscript ${ }^{*}$ refers to dimensional variables, $V^{*}=\mathrm{d} X^{*} / \mathrm{d} t^{*}$ is the velocity of the particle, $X^{*}$ is the position of the particle and $m_{p}$ is the mass of particle. 
We should note that Equation (2) does not incorporate compressibility of the particle and is valid in the long wavelength limit. The first term in the right-hand side of Equation (2) is the Stokes drag, where $\mu$ is the dynamic viscosity of the fluid, and $R$ is the particle radius; next two terms are the added mass force and history force, where $m_{f}$ is the mass of displaced fluid, and $v$ is the kinematic viscosity of the fluid. These three forces represent unsteady forces acting on an isolated particle in a viscous fluid, which have been extensively studied for rigid (Ardekani \& Rangel, 2006; Lovalenti \& Brady, 1993) and self-propelled (Wang \& Ardekani, 2012) particles. The corresponding dimensionless form of Equation (2) is

$$
S t\left(\frac{2 \gamma+1}{9}\right) \frac{\mathrm{d} V}{\mathrm{~d} t}+V+\sqrt{S t} \int_{0}^{t} \mathrm{~d} \tau \frac{\mathrm{d} V / \mathrm{d} \tau}{[\pi(t-\tau)]^{1 / 2}}=\sin (k X)
$$

where $t=t^{*}\left(V^{+} / R\right)$ is the dimensionless time, $V=V^{*} / V^{+}$is the dimensionless velocity, $X=X^{*} / R$ is the dimensionless position and $\gamma=\rho_{p} / \rho_{f}$ is the ratio of the particle density $\rho_{p}$ to the fluid density $\rho_{f}$. The characteristic velocity scale $V^{+}=\frac{3 \tilde{V} E_{a c}^{*} K|G|}{6 \pi \mu R}$ is derived from the balance between the acoustic force and Stokes drag, where $|G|$ is the magnitude of $G$. In this work, we consider neutrally buoyant particles, $\gamma=1$. The Stokes number in Equation (3) is defined as $S t=V^{+} t_{v} / R=V^{+} R / v$, and the dimensionless wave number is $k=2 K R=4 \pi R / \lambda . t_{v}=R^{2} / v$ is the viscous damping time scale. For applications of acoustophoresis (Aboobaker et al., 2005; Augustsson et al., 2011; Li et al., 2015) (see Table 1), typical ranges of parameters are $S t \sim O\left(10^{-3}-10^{1}\right.$ ), and $k \sim O\left(10^{-2}-10^{0}\right)$. Unless otherwise stated, hereafter equations and associated parameters such as time, location, velocities and forces are expressed in dimensionless forms. We should note that the current work focuses on rigid particles, and an extension to deformable cells is of future interest.

We use a Laplace transform to solve Equation (3). For a particle starting from rest, the Laplace transform of Equation (3) becomes

$$
\left[S t\left(\frac{2 \gamma+1}{9}\right) s+1+\sqrt{S t \cdot s}\right] \hat{V}=\hat{\mathrm{f}}
$$

Table 1. Physical and dimensionless parameters for acoustophoresis.

\begin{tabular}{lcccc}
\hline & $R(\mu \mathrm{m})$ & $V^{+}(\mathrm{mm} / \mathrm{s})$ & $S t$ & $k$ \\
\hline $\begin{array}{l}\text { Tracer particle Augustsson et al. } \\
\text { (2011) }\end{array}$ & 2.5 & .7 & .0019 & .082 \\
$\begin{array}{l}\text { Hollow microspheres (potters 60P18) } \\
\text { Setayeshgar, Lipsett, Koch, and }\end{array}$ & 9 & 9.5 & .086 & .11 \\
$\begin{array}{l}\text { Nobes (2015) } \\
\text { MCF-7 breast cancer Li et al. (2015), }\end{array}$ & $8-12$ & $38-75$ & $.30-1.0$ & $.50-.75$ \\
$\begin{array}{l}\text { Moon et al. (2011) } \\
\text { WBCs Li et al. (2015) }\end{array}$ & 6 & 21 & .12 & .37 \\
\hline
\end{tabular}


where represents variables in the Laplace space, and $s$ is the Laplace variable. The temporal evolution of particle velocity is calculated using the convolution $V=\int_{0}^{t} \mathrm{f}(t-\tau) g(\tau) \mathrm{d} \tau=\mathrm{f} * g$, and

$$
g=L^{-1}\left\{\frac{1}{S t\left(\frac{2 \gamma+1}{9}\right) s+1+\sqrt{S t \cdot s}}\right\},
$$

where $L^{-1}\{\cdot\}$ is the Laplace inverse operator.

\subsection{Equation of motion for a pair of interacting spherical particles}

The arbitrary motion of two particles in a viscous fluid can be decomposed into the motion along and perpendicular to their line of centres (Kim \& Karrila, 1991). We use the coordinate system shown in Figure 1(b), where $z$ and $\rho$ are coordinates measured along and perpendicular to the particles' line of centres, respectively.

In order to include the effect of hydrodynamic interaction between the particles, the method of reflections (Happel \& Brenner, 1983) is used to satisfy

(a)

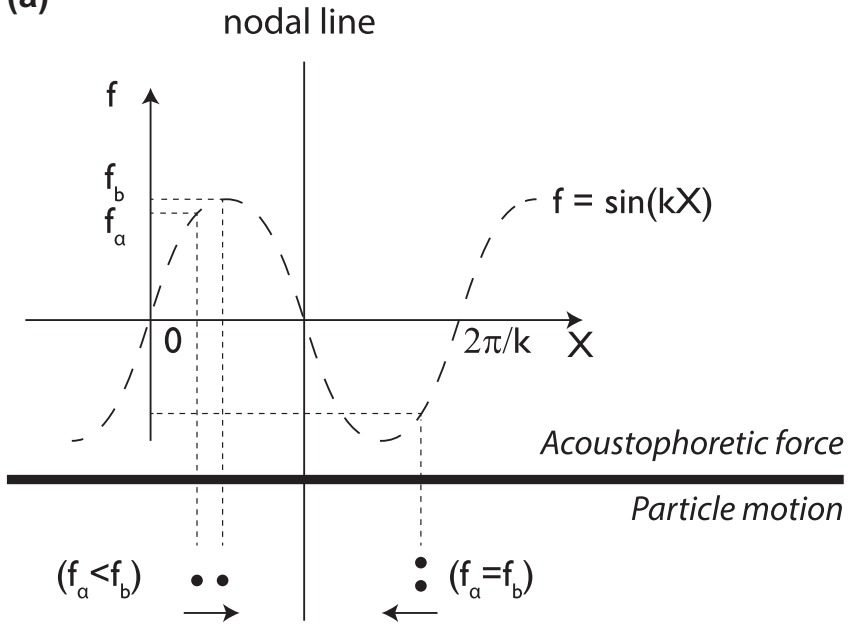

(b)

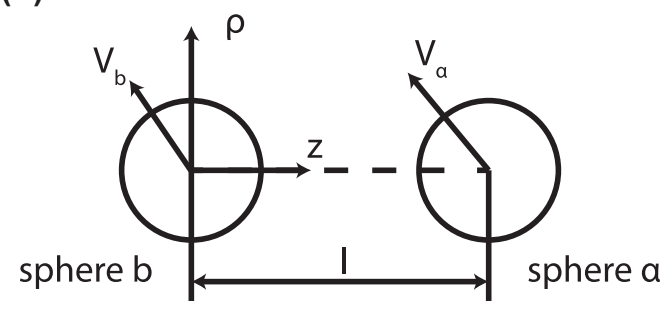

Figure 1. (a) Acoustic force f acting on two particles moving side by side or in tandem; (b) schematic of the problem and coordinate system. 
boundary conditions on both particles. The detailed derivation of the hydrodynamic force acting on two interacting particles is provided in Appendix 1. The equation of motion for particle $a$ in Laplacian space is written as

$$
\frac{2 \gamma}{9} S t_{a}\left(s \hat{\mathbf{V}}_{a}\right)=-\hat{\Lambda}_{a}\left(\frac{\hat{V}_{a \rho}-\hat{A}_{b} \hat{V}_{b \rho}}{1-\hat{A}_{a} \hat{A}_{b}} \mathbf{e}_{\rho}+\frac{\hat{V}_{a z}-\hat{B}_{b} \hat{V}_{b z}}{1-\hat{B}_{a} \hat{B}_{b}} \mathbf{e}_{z}\right)+\hat{\mathbf{f}}_{a}
$$

where $\mathbf{V}_{a}=V_{a \rho} \mathbf{e}_{\rho}+V_{a z} \mathbf{e}_{z}$, and $\hat{\mathbf{f}}_{a}$ is the acoustic force acting on particle $a$; $S t_{a}=V_{a}^{+} R_{a} / v$ and $R_{a}$ are the Stokes number and radius for particle $a$, respectively. Similarly, the equation of motion for particle $b$ is

$$
\frac{2 \gamma}{9} \operatorname{St}_{b}\left(s \hat{\mathbf{V}}_{b}\right)=-\hat{\Lambda}_{b}\left(\frac{\hat{V}_{b \rho}-\hat{A}_{a} \hat{V}_{a \rho}}{1-\hat{A}_{a} \hat{A}_{b}} \mathbf{e}_{\rho}+\frac{\hat{V}_{b z}-\hat{B}_{a} \hat{V}_{a z}}{1-\hat{B}_{a} \hat{B}_{b}} \mathbf{e}_{z}\right)+\hat{\mathbf{f}}_{b} .
$$

$\hat{\Lambda}_{a}, \hat{\Lambda}_{b}, \hat{A}_{a}, \hat{A}_{b}, \hat{B}_{a}$ and $\hat{B}_{b}$ are functions of the Laplace variable $s$, and their exact forms are given in Appendix 1.

Consider two identical particles side by side (see Figure 1(a)) moving along $\rho$ direction and $V_{a z}=V_{b z}=0, V_{a \rho}=V_{b \rho}=V$. For two identical particles $(a=b)$, we have $R_{a}=R_{b}=R, \hat{A}_{a}=\hat{A}_{b}=\hat{A}, \hat{B}_{a}=\hat{B}_{b}=\hat{B}$, and $\hat{\Lambda}_{a}=\hat{\Lambda}_{b}=\Lambda$. The equations of motion for the two particles are the same

$$
\frac{2 \gamma}{9} \tilde{s} \hat{V}=-\hat{\Lambda}\left(\frac{\hat{V}}{1+\hat{A}}\right)+\hat{\mathrm{f}}
$$

where $\tilde{s}=S t \cdot s$. The velocity of the particle can be obtained as

$$
V=\mathrm{f} * L^{-1}\left\{\frac{1+\hat{A}}{\frac{2 \gamma}{9} \tilde{s}(1+\hat{A})+\hat{\Lambda}}\right\} .
$$

Next, we consider two identical particles in tandem moving along $\mathrm{z}$ axis $\left(V_{a \rho}=V_{b \rho}=0\right)$. Since the acoustic force acting on each particle depends on its instantaneous position, the equations of motion for particles $a$ and $b$ are different

$$
\frac{2 \gamma}{9} \tilde{s} \hat{V}_{a}=-\hat{\Lambda}\left(\frac{\hat{V}_{a}-\hat{B} \hat{V}_{b}}{1-\hat{B}^{2}}\right)+\hat{\mathrm{f}}_{a}
$$

and

$$
\frac{2 \gamma}{9} \tilde{s} \hat{V}_{b}=-\hat{\Lambda}\left(\frac{\hat{V}_{b}-\hat{B} \hat{V}_{a}}{1-\hat{B}^{2}}\right)+\hat{\mathrm{f}}_{b}
$$


The temporal evolution of particle velocities is given as

$$
\begin{gathered}
V_{a}=L^{-1}\left\{\frac{1}{2}\left(\frac{\hat{\mathrm{f}}_{a}-\hat{\mathrm{f}}_{b}}{\frac{2 \gamma}{9} \tilde{s}+\frac{\hat{\Lambda}}{1-\hat{B}}}+\frac{\hat{\mathrm{f}}_{a}+\hat{\mathrm{f}}_{b}}{\frac{2 \gamma}{9} \tilde{s}+\frac{\hat{\Lambda}}{1+\hat{B}}}\right)\right\}, \\
V_{b}=L^{-1}\left\{-\frac{1}{2}\left(\frac{\hat{\mathrm{f}}_{a}-\hat{\mathrm{f}}_{b}}{\frac{2 \gamma}{9} \tilde{s}+\frac{\hat{\Lambda}}{1-\hat{B}}}-\frac{\hat{\mathrm{f}}_{a}+\hat{\mathrm{f}}_{b}}{\frac{2 \gamma}{9} \tilde{s}+\frac{\hat{\Lambda}}{1+\hat{B}}}\right)\right\} .
\end{gathered}
$$

\section{Results and discussions}

\subsection{Motion of an isolated particle}

The motion of a solitary particle strongly depends on its Stokes number, $S t=\frac{2 R^{3} \rho_{f} E_{a c}^{*} K G}{3 \pi \mu^{2}}$. For a given acoustic resonator system, the relevant Stokes number is proportional to the particle volume. First, we quantify the unsteady effects on the particle motion by comparing the results to the solution of the steady Stokes theory. The steady Stokes theory, widely used in the literature (Augustsson et al., 2011; Bruus, 2012; Ding et al., 2014), corresponds to a quasisteady state regime, where the acoustic force is balanced with the Stokes drag, and Equation (3) is simplified to $V=\sin (k X)$. When $S t=.1$, the particle trajectory is close to the one predicted by the steady Stokes theory which neglects the unsteady inertial forces (see Figure 2(a)). On the other hand, the trajectory of a particle at $S t=1$ (corresponding to $10 \mu \mathrm{m}$ particle) deviates from the quasi-steady solution. Interestingly, instead of approaching the pressure nodal line before coming to rest at $k X / \pi=1$ as a small particle would do, a particle at $S t=10$ passes the pressure nodal line at the first encounter due to the strong unsteady inertial force. The particle velocity (see Figure $2(\mathrm{~b})$ ) for $S t=10$ remains positive at the pressure nodal line. This positive velocity drives the particle away from the nodal line. Eventually, the particle returns to the nodal line due to the acoustic force. Note that, the dimensionless time at which the particle stops at the pressure nodal line increases with the particle Stokes number and consequently particle radius (see Figure 2(a)).

The unsteady inertial force reduces the maximum velocity of the particle $V_{\max }$. The relationship between $S t$ and $V_{\max }$ is given in Figure 2(c). In a quasi-steady regime $(S t=0)$, the particle maximum velocity occurs at $k X / \pi=1 / 2$. At large $S t$, the location at which the particle reaches its maximum velocity deviates from $k X / \pi=1 / 2$, resembling a 'phase lag' (see Figure 2(b)). Furthermore, we examine the effect of $S t$ on the forces acting on the particle: Stokes drag $(V)$, history force $\left(\sqrt{S t} \int_{0}^{t} \mathrm{~d} \tau \frac{\mathrm{d} V / \mathrm{d} \tau}{[\pi(t-\tau)]^{1 / 2}}\right)$, and added mass force $\left(\frac{S t}{9} \frac{\mathrm{d} V}{\mathrm{~d} t}\right)$. When $S t=0$, the Stokes drag balances the acoustic force. At $S t=10$, the magnitude of the added mass force is minute, but the history force is comparable to the Stoke drag thus it cannot be neglected (see Figure 2(d)). Therefore, the particle motion is 
(a)

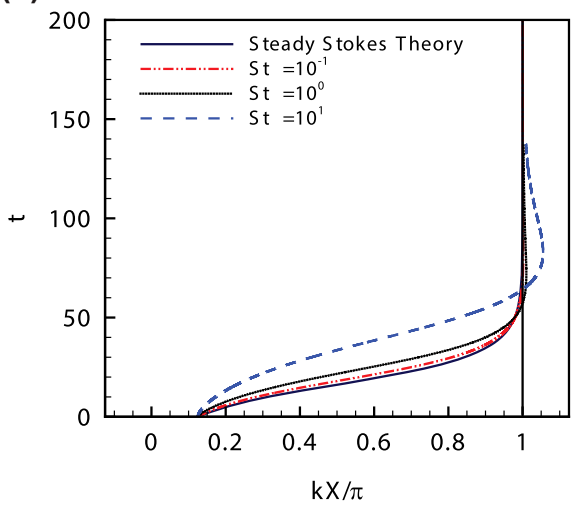

(b)

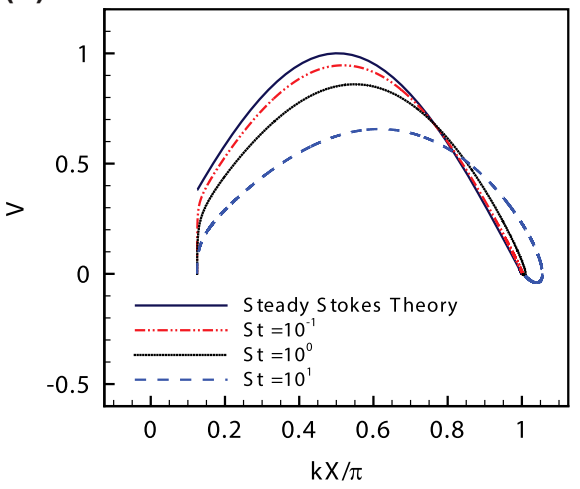

(c)

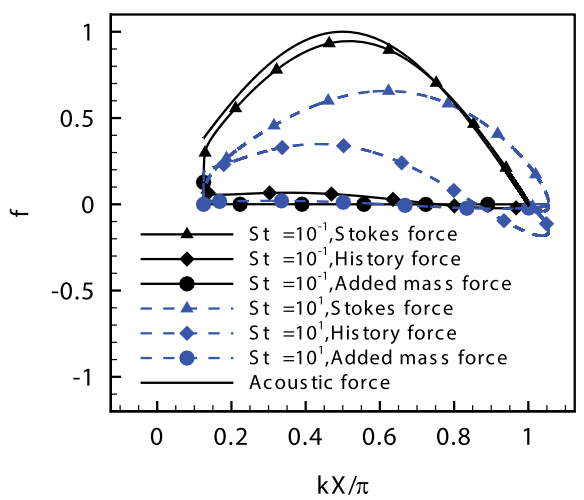

(d)

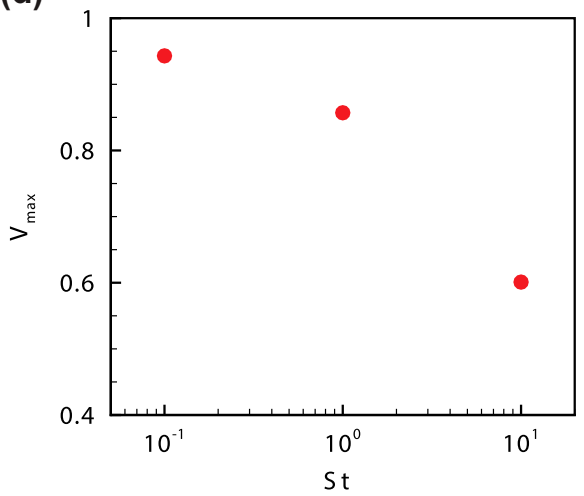

Figure 2. The motion of an isolated particle in an acoustic field in a viscous fluid: (a) time, (b) velocity and (c) forces are plotted as a function of particle's transverse position $X(t)$, where the solid straight line in (a) indicates the nodal line of the acoustic system. (d) The maximum particle velocity is plotted as a function of $S t$.

Note: The particle starts from rest at $k X / \pi=1 / 8$, for $k=.1$.

significantly affected by the history force in the presence of an unsteady inertial effect.

In addition, we investigate the motion of a solitary particle under different dimensionless wave numbers $k$ in the presence of unsteady inertial effects. We note that in the quasi-steady regime, the particle velocity is independent of $k$. However, the particle velocity is reduced for larger wave numbers when unsteady inertial effects are included (see Figure 3(a)). By comparing unsteady forces for different wave numbers in Figure 3(b), we find that the history force is larger for large wave numbers.

\subsection{Particle motion due to pair hydrodynamic interaction}

We can investigate hydrodynamic forces acting on a particle in a dilute suspension in the presence of unsteady inertial effects where pair particle interaction is dominant. The particle motion (black filled circle in Figure 4(a)) in the presence of a second particle (dashed unfilled circle) is obtained for 
(a)

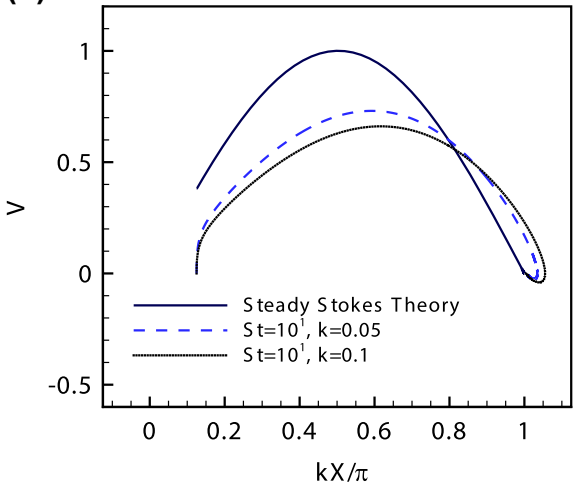

(b)

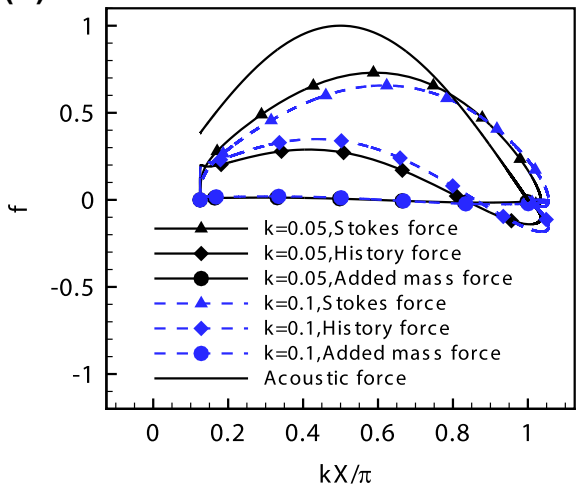

Figure 3. The effect of wave number $k$ on the motion of an isolated particle in an acoustic field in a viscous fluid: (a) velocity and (b) forces are plotted as a function of particle's transverse position $X$.

Note: The particle starts from rest at $k X / \pi=1 / 8$, for $S t=10$.

a side-by-side configuration, denoted as ' $\mathrm{A}$ ' and two in-tandem configurations (' $\mathrm{B}$ ' and ' $\mathrm{C}$ '). Configuration ' $\mathrm{B}$ ' corresponds to the case in which the second particle is in front of the main particle moving towards the nodal line, while in configuration ' $\mathrm{C}$ ', the second particle is behind the main particle. Figure 4(a) compares the motion of the main particle in three different configurations (' $A$ ', ' $\mathrm{B}$ ', and ' $\mathrm{C}$ ') against the motion of a solitary particle in absence of particle-particle hydrodynamic interaction. Two in-tandem particles eventually collide near the nodal line, but we should note that the method of reflections used here holds for large particle distances (Ardekani \& Rangel, 2006). Therefore, in Figure 4, we show the particle trajectory when the distance between particles is larger than 4 radii $(\epsilon \leq 1 / 4)$, where $\epsilon$ is the inverse of dimensionless distance between particles. The solid circle and triangle in Figure 4(a) show the final calculated positions for configurations ' $\mathrm{B}$ ' and 'C', respectively. The trajectories of both particles in configurations ' $\mathrm{B}$ ' and ' $\mathrm{C}$ ' are given in Figure 4(b).

Next, we investigate the effect of the hydrodynamic interaction on the unsteady forces in configuration ' $\mathrm{C}$ ' by comparing the results to the ones for the solitary particle. It is well known that the hydrodynamic interaction reduces the Stokes drag in the quasi-steady case (Kim \& Karrila, 1991). Similarly, the hydrodynamic interaction reduces the Stokes force in the presence of unsteady inertial effects (see Figure 4(c)). On the other hand, the history force is enhanced in the presence of the neighbouring particle. The strength of the hydrodynamic interaction is influenced by the distance between two particles. Particle velocity for different Stokes number and particle initial distance is plotted in Figure 4(d). The particle velocity is enhanced when particle hydrodynamic interaction is included and note it is reduced when unsteady inertial effects are considered. In the presence of unsteady inertial effect, the particle's maximum velocity decreases as the dimensionless wave number increases (see Figure 5). 
(a)

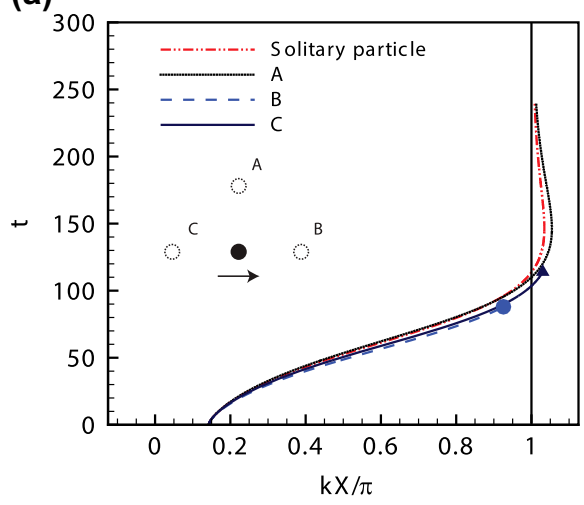

(b)

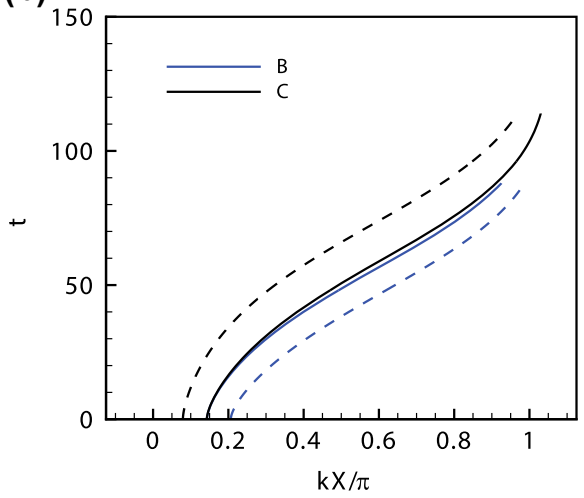

(c)

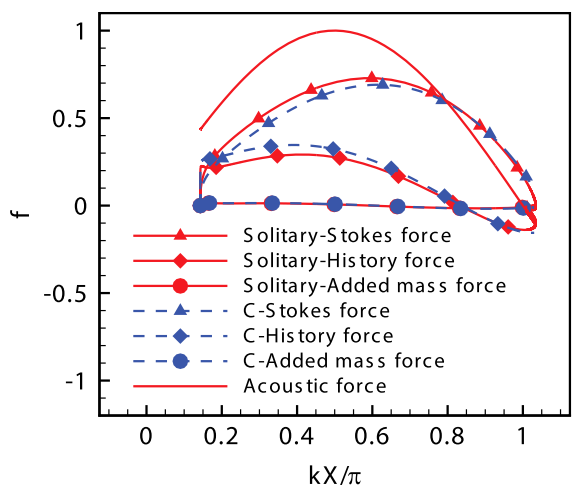

(d)

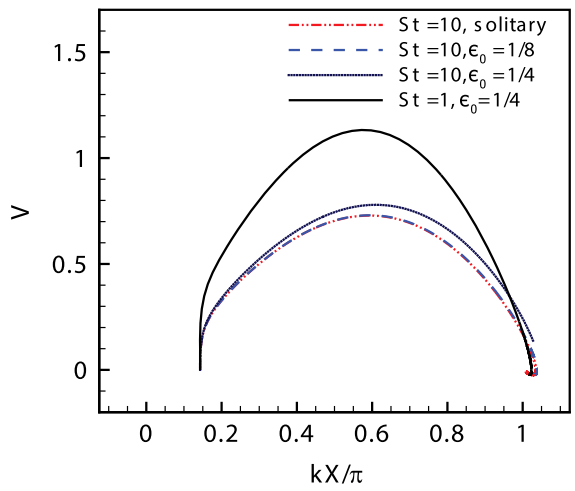

Figure 4. (a) The particle motion is affected by the particle-particle hydrodynamic interaction. (b) Trajectory of both particles is shown for two different in-tandem configurations; the solid and dashed lines correspond to the main particle and the neighbouring particle, respectively. (c) The effect of particle-particle hydrodynamic interaction on unsteady forces acting on the particles in configuration ' $C$ '. (d) The effect of distance between particles on their motion.

Notes: The wave number is $k=.05$. For (a), (b), and (c), the initial distance $\epsilon_{0}=1 / 4$ and $S t=10$. The particles start from rest at $k X / \pi=1 / 7$.

\subsection{Tilted-angle standing surface acoustic waves}

We consider particles that are exposed to a uniform flow and experience a standing acoustic field along the $X$ axis (see Figure 6(a)) to analyse a taSSAW separation approach. The established pressure nodal and antinodal lines have an angle with respect to the imposed flow direction, and the total driving force is $\mathbf{f}=\mathrm{f}_{F} \boldsymbol{e}_{\theta}+\mathrm{f}_{a c} \boldsymbol{e}_{X}$, where $\mathrm{f}_{F}$ is the magnitude of the force imposed by the uniform flow, $\boldsymbol{e}_{\theta}$ is the unit vector along the flow direction and $\theta$ indicates the angle between the flow direction and $X$ axis. Note that for a typical taSSAW (Li et al., 2015), $\frac{\mathrm{f}_{F}}{\mathrm{f}_{a c}} \sim O(1)$ and the angle $\theta$ remains small $\left(\sim 5^{\circ}\right)$. The combination of hydrodynamic and acoustic forces affects the particle displacement $\Delta X$ and $\Delta Y$.

To understand the role of unsteady inertial forces on the trajectory of a solitary particle, we compare our results to the trajectory predicted by the steady Stokes theory $(S t=0)$. At $S t=1$, the unsteady inertial forces reduce $\Delta Y$ for the same 


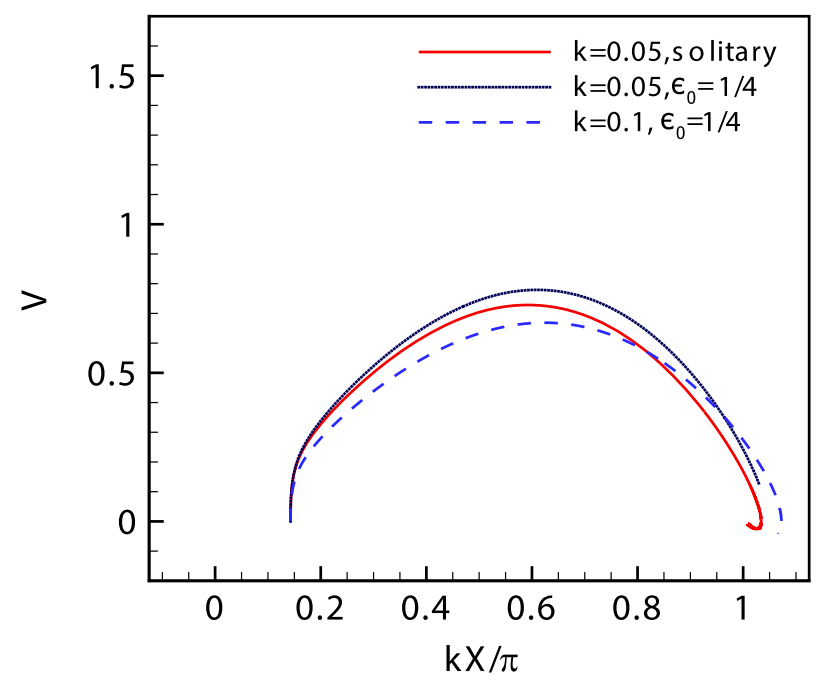

Figure 5. The effect of dimensionless wave number $k$ on the interacting particles. Note: The particles start from rest at $k X / \pi=1 / 7$, for $S t=10$.

(a)

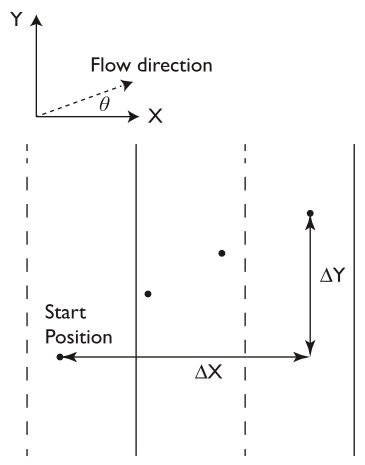

(b)

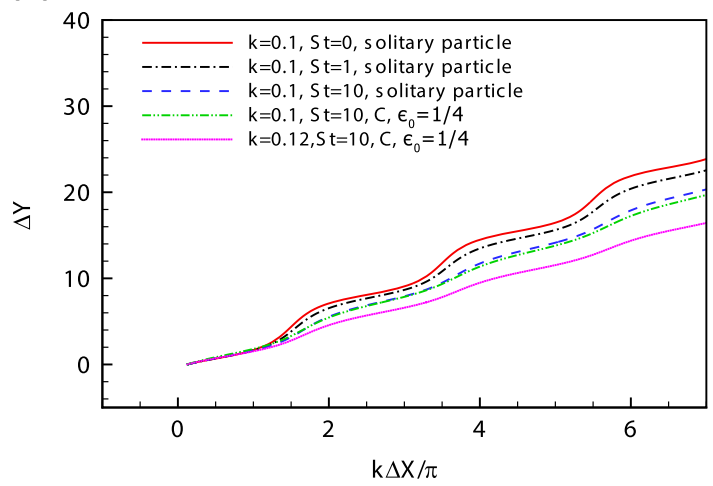

Figure 6. (a) Schematic of the taSSAW-based particle motion. The pressure nodal and antinodal lines are indicated as solid and dashed lines, respectively. (b) taSSAW-based particle motion can be significantly modified by unsteady inertial effects $(S t)$, particles hydrodynamic interaction, and dimensionless wave number $(k)$.

Notes: The acoustic force is along $X$ axis, and the angle $\theta$ between the flow direction and $X$ axis is $5^{\circ}$. The imposed force is $\mathrm{f}_{F}=1.5$, and the particles start from rest at $k \Delta X / \pi=1 / 8$.

displacement $\Delta X$. A larger Stokes number leads to a larger deviation from the quasi-steady case (see Figure 6(b)). The lateral displacement of particles reduces with Stokes number, resulting from reduction of particle velocities $V_{X}$ and $V_{Y}$ for large values of Stokes number (see Figure 7). Similarly, the lateral displacement of particle reduces with the dimensionless wave number (see Figure 7).

Finally, we investigate the effect of the particles' hydrodynamic interactions. At $S t=10$, we compare the particle trajectory between solitary case and intandem configuration ' $\mathrm{C}$ ' in Figure 6(b). The particle lateral displacement $\Delta Y$ for the in-tandem configuration is smaller than the solidary particle. The particle 
(a)

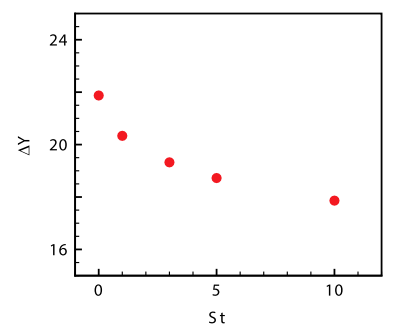

(b)

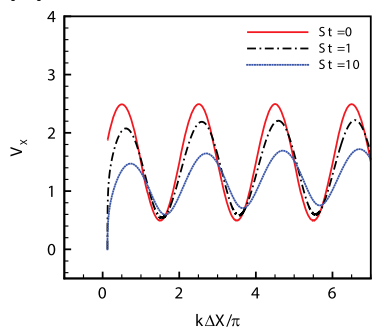

(c)

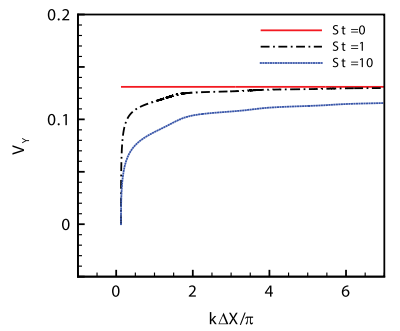

Figure 7. Effect of unsteady inertial forces on (a) lateral displacement $\Delta Y$, (b) velocity $V_{X}$ and (c) velocity $V_{Y}$ at $k \Delta X / \pi=6$.

Notes: The parameters corresponding to taSSAW are $\theta=5^{\circ}, k=.1, \mathrm{f}_{F}=1.5$, and the particles start from rest at $k \Delta X / \pi=1 / 8$.

(a)

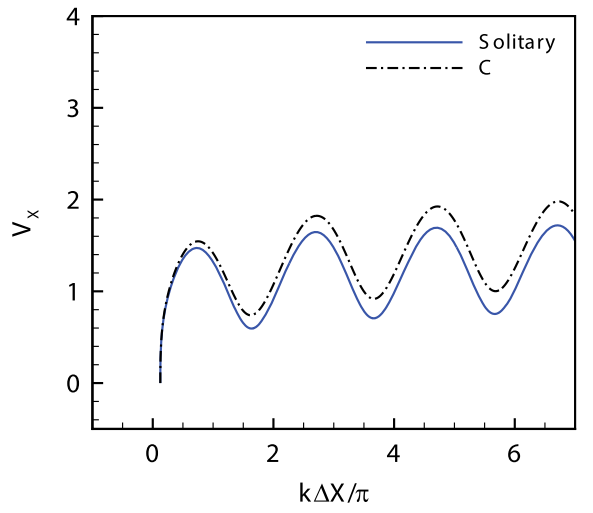

(b)

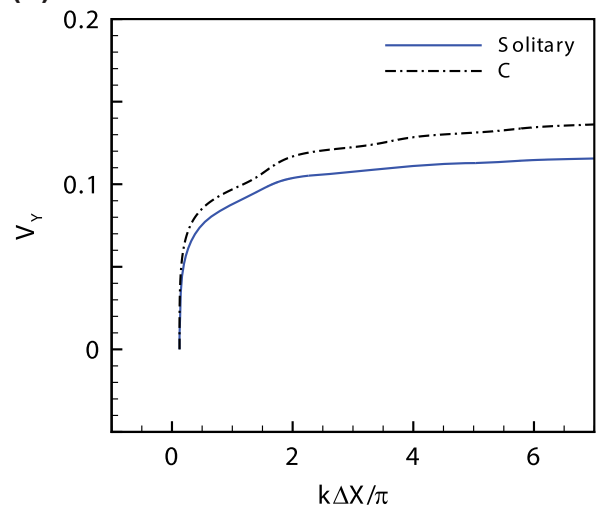

Figure 8. Effect of particle hydrodynamic interaction on (a) velocity component $V_{X}$, and (b) velocity component $V_{Y}$.

Notes: The initial distance between particles is $\epsilon_{0}=1 / 4$, and $S t=10$. The parameters corresponding to taSSAW are $\theta=5^{\circ}, k=.1, \mathrm{f}_{\mathrm{F}}=1.5$, and the particles start from rest at $k \Delta X / \pi=1 / 8$.

velocity is on the other hand larger if the hydrodynamic interaction is included (Figure 8).

\section{Conclusions}

In this work, we investigated effects of unsteady inertial forces on the particle motion in an acoustic standing wave field. We considered the effect of particleparticle interaction in the presence of unsteady inertial effects. The hydrodynamic interaction reduces the Stokes force and enhances the history force, leading to larger particle velocities. In taSSAW-based separation designs, we illustrated that the particle trajectory significantly deviates from the prediction of the steady Stokes theory due to both unsteady inertial effects and hydrodynamic interactions. Both unsteady inertial forces and hydrodynamic interactions reduce the particle lateral displacement $\Delta Y$. Such considerations will be important for the design of optimal acoustic separation devices. 


\section{Disclosure statement}

No potential conflict of interest was reported by the authors.

\section{Funding}

This work is partially supported by NSF [grant number CBET-1445955].

\section{ORCID}

A. M. Ardekani (D) http://orcid.org/0000-0003-3301-3193

\section{References}

Aboobaker, N., Blackmore, D., \& Meegoda, J. (2005). Mathematical modeling of the movement of suspended particles subjected to acoustic and flow fields. Applied Mathematical Modelling, 29, 515-532.

Allen, J. S., Kobayashi, M. H., \& Coimbra, C. F. M. (2006). History effects on the viscous motion of acoustically forced particles. Applied Physics Letters, 88, 214106-214106.

Ardekani, A. M., \& Rangel, R. H. (2006). Unsteady motion of two solid spheres in Stokes flow. Physics of Fluids, 18, 103306.

Augustsson, P., Barnkob, R., Wereley, S. T., Bruus, H., \& Laurell, T. (2011). Automated and temperature-controlled micro-PIV measurements enabling long-term-stable microchannel acoustophoresis characterization. Lab Chip, 11, 4152-4164.

Bruus, H. (2012). Acoustofluidics 7: The acoustic radiation force on small particles. Lab Chip, $12,1014-1021$.

Coakley, W. T., Whitworth, G., Grundy, M. A., Gould, R. K., \& Allman, R. (1994). Ultrasonic manipulation of particles and cells. Ultrasonic separation of cells. Bioseparation, 4, 73-83.

Ding, X., Peng, Z., Lin, S.-C. S., Geri, M., Li, S., Li, P., ... Huang, T. J. (2014). Cell separation using tilted-angle standing surface acoustic waves. Proceedings of the National Academy of Sciences, 111, 12992-12997.

Friend, J., \& Yeo, L. Y. (2011). Microscale acoustofluidics: Microfluidics driven via acoustics and ultrasonics. Reviews of Modern Physics, 83, 647-704.

Guo, F., Mao, Z., Chen, Y., Xie, Z., Lata, J. P., Li, P., ... Dao, Ming (2016). Three-dimensional manipulation of single cells using surface acoustic waves. Proceedings of the National Academy of Sciences, 113, 1522-1527.

Happel, J., \& Brenner, H. (1983). Low Reynolds number hydrodynamics: With special applications to particulate media. Vol. 1, Springer Science \& Business Media.

Johnson, D. A., \& Feke, D. L. (1995). Methodology for fractionating suspended particles using ultrasonic standing wave and divided flow fields. Separation and Technology, 5, 251-258.

Kim, S., \& Karrila, S. J. (1991). Microhydrodynamics: Principles and selected applications. Boston, MA: Butterworth-Heinemann.

King, L. V. (1934). On the acoustic radiation pressure on spheres. Proceedings of the Royal Society A, 147, 212-240.

Laurell, T., Petersson, F., \& Nilsson, A. (2007). Chip integrated strategies for acoustic separation and manipulation of cells and particles. Chemical Society Reviews, 36, 492-506.

Li, P., Mao, Z., Peng, Z., Zhou, L., Chen, Y., Huang, P.-H., ... Dao, M. (2015). Acoustic separation of circulating tumor cells. Proceedings of the National Academy of Sciences, 112, 4970-4975. 
Lovalenti, P. M., \& Brady, J. F. (1993). The hydrodynamic force on a rigid particle undergoing arbitrary time-dependent motion at small Reynolds number. Journal of Fluid Mechanics, 256, 561-605.

Maxey, M. R., \& Riley, J. J. (1983). Equation of motion for a small rigid sphere in a nonuniform flow. Physics of Fluids, 26, 883-889.

Moon, H.-S., Kwon, K., Kim, S.-I., Han, H., Sohn, J., Lee, S., \& Jung, H.-I (2011). Continuous separation of breast cancer cells from blood samples using multi-orifice flow fractionation (moff) and dielectrophoresis (dep). Lab Chip, 11, 1118-1125.

Plaks, V., Koopman, C. D., \& Werb, Z. (2013). Circulating tumor cells. Science, 341, 11861188.

Setayeshgar, A., Lipsett, M. G., Koch, C. R., \& Nobes, D. S. (2015). Particle motion in a macroscale, multiwavelength acoustic field. Journal of Fluids Engineering, 137, 011302.

Settnes, M., \& Bruus, H. (2012). Forces acting on a small particle in an acoustical field in a viscous fluid. Physical Review E, 85, 016327.

Temkin, S. (2005). Suspension acoustics: An introduction to the physics of suspensions. New York, NY: Cambridge University Press.

Temkin, S., \& Leung, C.-M. (1976). On the velocity of a rigid sphere in a sound wave. Journal of Sound and Vibration, 49, 75-92.

Temkin, S. (1981). Elements of acoustics. New York, NY: Wiley.

Wang, S., \& Ardekani, A. M. (2012). Unsteady swimming of small organisms. The Journal of Fluid Mechanics, 702, 286-297.

Weiser, M. A. H., Apfel, R. E., \& Neppiras, E. A. (1984). Interparticle forces on red cells in a standing wave field. Acta Acustica United with Acustica, 56, 114-119.

Yeo, L. Y., \& Friend, J. R. (2009). Ultrafast microfluidics using surface acoustic waves. Biomicrofluidics, 3, 012002.

\section{Appendix 1. Derivation of hydrodynamic forces on two interacting particles}

For micrometer-sized acoustic particles $(\sim O(10 \mu \mathrm{m}))$ submerged in a fluid, the convective terms can be neglected (Maxey \& Riley, 1983), and the dimensionless governing equations for an unsteady Stokes flow can be written as,

$$
\begin{array}{r}
S t \frac{\partial \mathbf{u}}{\partial t}=-\nabla p+\nabla^{2} \mathbf{u}, \\
\nabla \cdot \mathbf{u}=0,
\end{array}
$$

where $\mathbf{u}$ is the flow velocity, and $p$ is the dynamic pressure. The particle in general undergoes translation and rotation, which can be considered separately due to the linearity of governing equations and boundary conditions. Here, we restrict our attention to the translational motion without rotation. For a particle pair in a quiescent fluid (see Figure 1), the boundary conditions are

$$
\begin{aligned}
\mathbf{u} & =\mathbf{V}_{a}, \text { onparticle } a \\
\mathbf{u} & =\mathbf{V}_{b}, \text { onparticle } b, \\
\left.\mathbf{u}\right|_{r \rightarrow \infty} & =0,
\end{aligned}
$$

where $r=\sqrt{\rho^{2}+z^{2}}$. To solve this boundary value problem, we use the method of reflections to approximately satisfy the boundary conditions on both particles. Since the governing equations and boundary conditions are linear, the flow velocity and pressure can be decomposed 


$$
\begin{aligned}
& \mathbf{u}=\mathbf{u}^{(1)}+\mathbf{u}^{(2)}+\mathbf{u}^{(3)}+\mathbf{u}^{(4)}+\mathbf{u}^{(5)}+\ldots, \\
& p=p^{(1)}+p^{(2)}+p^{(3)}+p^{(4)}+p^{(5)}+\ldots,
\end{aligned}
$$

where $\left(\mathbf{u}^{(i)}, p^{(i)}\right)$ satisfy the equations of motion associated with an isolated particle and vanish at infinity. For example, $\left(\mathbf{u}^{(1)}, p^{(1)}\right)$ satisfy the boundary condition on particle $a, \mathbf{V}^{(1)}=\mathbf{V}_{a}$. The reflection of this field from particle $b$ can be defined by the boundary condition on particle $b, \mathbf{V}^{(2)}=\mathbf{V}_{b}-\left.\mathbf{u}^{(1)}\right|_{b}$, where $\left.\mathbf{u}^{(1)}\right|_{b}$ is velocity $\mathbf{u}^{(1)}$ evaluated at the centre of particle $b$. The third-order term can be found through the reflection of $\mathbf{u}^{(2)}$ from particle $a$ and its boundary condition on particle $a, \mathbf{V}^{(3)}=-\left.\mathbf{u}^{(2)}\right|_{a}$. Again, the next order term can be calculated by defining the boundary condition on particle $b, \mathbf{V}^{(4)}=-\left.\mathbf{u}^{(3)}\right|_{b}$. Similarly, higher order terms are calculated to satisfy boundary conditions on the surface of both particles with the required accuracy. Once $\mathbf{u}^{(i)}$ is computed, the drag force due the flow field $\mathbf{u}^{(i)}$ can be written as

$$
\mathbf{F}^{(i)}=-\frac{S t}{9} \frac{\mathrm{d} \mathbf{V}^{(i)}}{\mathrm{d} t}-\mathbf{V}^{(i)}-\sqrt{S t} \int_{0}^{t} \mathrm{~d} \tau \frac{\mathrm{d} \mathbf{V}^{(i)} / \mathrm{d} \tau}{[\pi(t-\tau)]^{1 / 2}},
$$

or in Laplace space $(i=1,2,3, \ldots)$,

$$
\begin{aligned}
\hat{\mathbf{F}}^{(i)} & =-\left(1+\sqrt{\tilde{s}}+\frac{\tilde{s}}{9}\right) \hat{\mathbf{V}}^{(i)}, \\
& =-\hat{\Lambda} \hat{\mathbf{V}}^{(i)},
\end{aligned}
$$

where $\tilde{s}=S t \cdot s$. The total force exerted on a given particle (e.g., particle $a$ ) can be obtained using the summation of the drag force generated by each flow field $\mathbf{u}^{(i)}$. Therefore, the total drag on particle $a$ can be calculated as

$$
\mathbf{F}_{a}=\mathbf{F}^{(1)}+\mathbf{F}^{(3)}+\mathbf{F}^{(5)}+\cdots
$$

where $\mathbf{F}^{(1)}$ is the unsteady force acting on an isolated particle due to flow field $\mathbf{u}^{(1)}$, and the sum of higher order terms accounts for the hydrodynamic interaction between two particles.

For the motion of an isolated spherical particle, the flow field generated by the particle is given by the Burger's solution that satisfies the unsteady Stokes equation with boundary condition $\mathbf{u}=(0,0, \delta(t))$ on the particle surface. By utilising the Burger's solution, we are able to analytically calculate $\mathbf{u}^{(i)}$ for each order.

The Burger's solution in a dimensional form can be written as

$$
\begin{aligned}
\hat{\mathbf{u}}_{B} & =\left(\mathbf{e}^{(3)} \cdot \nabla\right) \nabla \hat{\psi}-\mathbf{e}^{(3)} \nabla^{2} \hat{\psi}, \\
\hat{\psi}(r, \tilde{s}) & =\frac{Q_{1}}{r}+\frac{Q_{2}}{r} e^{-\sqrt{\tilde{s}} r},
\end{aligned}
$$

where $\mathbf{e}^{(3)}$ is the unit vector $(0,0,1), Q_{1}=\frac{3}{2 \tilde{s}}\left(1+\tilde{s}^{1 / 2}\right)+\frac{1}{2}, Q_{2}=-\frac{3}{2 \tilde{s}} e^{\sqrt{\tilde{s}}}$ and $\hat{\psi}=\hat{\psi}^{*} / R^{3}$. If particle $a$ moves with velocity $\mathbf{V}_{a}=V_{a \rho} \mathbf{e}_{\rho}+V_{a z} \mathbf{e}_{z}, \hat{\mathbf{V}}^{(2)}$ can be calculated using Equations (A11) and (A12)

$$
\hat{\mathbf{V}}^{(2)}=\left(\hat{V}_{b \rho}-\hat{V}_{a \rho} \hat{A}_{a}\right) \mathbf{e}_{\rho}+\left(\hat{V}_{b z}-\hat{V}_{a z} \hat{B}_{a}\right) \mathbf{e}_{z},
$$


and $\hat{A}_{a}$ and $\hat{B}_{a}$ are given as

$$
\begin{aligned}
& \hat{A}_{a}=\left.\hat{A}(\tilde{s}, \epsilon)\right|_{\tilde{s}=\tilde{s}_{a}, \epsilon=\epsilon_{a}}=\frac{3 \epsilon^{3}}{2 \tilde{s}}\left[-1-\sqrt{\tilde{s}}-\frac{\tilde{s}}{3}+e^{\sqrt{\tilde{s}}\left(1-\epsilon^{-1}\right)}\left(1+\frac{\sqrt{\tilde{s}}}{\epsilon}+\frac{\tilde{s}}{\epsilon^{2}}\right)\right], \\
& \hat{B}_{a}=\left.\hat{B}(\tilde{s}, \epsilon)\right|_{\tilde{s}=\tilde{s}_{a}, \epsilon=\epsilon_{a}}=\frac{3 \epsilon^{3}}{\tilde{s}}\left[1+\sqrt{\tilde{s}}+\frac{\tilde{s}}{3}-e^{\sqrt{\tilde{s}}\left(1-\epsilon^{-1}\right)}\left(1+\frac{\sqrt{\tilde{s}}}{\epsilon}\right)\right],
\end{aligned}
$$

where $\epsilon_{a}=R_{a} / l, \tilde{s}_{a}=S t_{a} \cdot s, S t_{a}=V_{a}^{+} R_{a} / v$ and $R_{a}$ is the radius of particle $a$.

Higher order velocity fields are given as

$$
\begin{aligned}
\hat{\mathbf{V}}^{(3)}= & \left(-\hat{V}_{b \rho} \hat{A}_{b}+\hat{V}_{a \rho} \hat{A}_{a} \hat{A}_{b}\right) \mathbf{e}_{\rho}+\left(-\hat{V}_{b z} \hat{B}_{b}+\hat{V}_{a z} \hat{B}_{a} \hat{B}_{b}\right) \mathbf{e}_{z} \\
\hat{\mathbf{V}}^{(5)}= & \left(-\hat{V}_{b \rho} \hat{A}_{b}+\hat{V}_{a \rho} \hat{A}_{a} \hat{A}_{b}\right) \hat{A}_{a} \hat{A}_{b} \mathbf{e}_{\rho}+\left(-\hat{V}_{b z} \hat{B}_{b}+\hat{V}_{a z} \hat{B}_{a} \hat{B}_{b}\right) \hat{B}_{a} \hat{B}_{b} \mathbf{e}_{z} \\
\cdot & \\
\cdot & \\
\hat{\mathbf{V}}^{(2 n+1)}= & \left(-\hat{V}_{b \rho} \hat{A}_{b}+\hat{V}_{a \rho} \hat{A}_{a} \hat{A}_{b}\right)\left(\hat{A}_{a} \hat{A}_{b}\right)^{n-1} \mathbf{e}_{\rho} \\
& +\left(-\hat{V}_{b z} \hat{B}_{b}+\hat{V}_{a z} \hat{B}_{a} \hat{B}_{b}\right)\left(\hat{B}_{a} \hat{B}_{b}\right)^{n-1} \mathbf{e}_{z} \cdot(n \geq 1)
\end{aligned}
$$

and total force $\hat{\mathbf{F}}_{a}$ is

$$
\begin{aligned}
\hat{\mathbf{F}}_{a}= & \hat{\mathbf{F}}^{(1)}+\hat{\mathbf{F}}^{(3)}+\hat{\mathbf{F}}^{(5)}+\ldots \\
= & -\hat{\Lambda}_{a}\left(\hat{\mathbf{V}}^{(1)}+\hat{\mathbf{V}}^{(3)}+\hat{\mathbf{V}}^{(5)}+\ldots\right) \\
= & -\hat{\Lambda}_{a} \hat{V}_{a \rho}\left(1+\hat{A}_{a} \hat{A}_{b}+\left(\hat{A}_{a} \hat{A}_{b}\right)^{2}+\ldots\right) \mathbf{e}_{\rho} \\
& +\hat{\Lambda}_{a} \hat{V}_{b \rho} \hat{A}_{b}\left(1+\hat{A}_{a} \hat{A}_{b}+\ldots\right) \mathbf{e}_{\rho}-\hat{\Lambda}_{a} \hat{V}_{a z}\left(1+\hat{B}_{a} \hat{B}_{b}+\left(\hat{B}_{a} \hat{B}_{b}\right)^{2}+\ldots\right) \mathbf{e}_{z} \\
& +\hat{\Lambda}_{a} \hat{V}_{b z} \hat{B}_{b}\left(1+\hat{B}_{a} \hat{B}_{b}+\ldots\right) \mathbf{e}_{z} \\
= & -\hat{\Lambda}_{a}\left(\frac{\hat{V}_{a \rho}-\hat{A}_{b} \hat{V}_{b \rho}}{1-\hat{A}_{a} \hat{A}_{b}} \mathbf{e}_{\rho}+\frac{\hat{V}_{a z}-\hat{B}_{b} \hat{V}_{b z}}{1-\hat{B}_{a} \hat{B}_{b}} \mathbf{e}_{z}\right)
\end{aligned}
$$

where the term in the right-hand side of Equation (A18) represents the total drag force, incorporating the hydrodynamic force due to the particle motion as well as the hydrodynamic interaction caused by the neighbouring particle. 